\title{
ANTI-SNAKE VENOM: USE AND ADVERSE REACTION IN A SNAKE BITE STUDY CLINIC IN BANGLADESH
}

\author{
AMIN MR (1), MAMUN SMH (2), RASHID R (3), RAHMAN M (4), GHOSE A (5), \\ SHARMIN S (6), RAHMAN MR (7), FAIZ MA (8)
}

(1) Consultant of Medicine, Hathazari Upazilla Health Complex, Chittagong, Bangladesh; (2) Assistant Professor of Cardiology, Chittagong Medical College Hospital, Chittagong, Bangladesh; (3) Radiologist, Chittagong General Hospital, Chittagong, Bangladesh; (4) Assistant Professor of Gastroenterology, Shahabuddin Medical College, Dhaka, Bangladesh; (5) Resident Medical Officer, Chittagong General Hospital, Chittagong, Bangladesh; (6) Department of Cardiology, Institute of Post-graduate Medicine and Research, IPGMR, Dhaka, Bangladesh; (7) Professor of Medicine, Begum Khaleda Zia Medical College, Dhaka, Bangladesh; (8) Professor of Medicine, Dhaka Medical College, Dhaka, Bangladesh.

\begin{abstract}
Snakebites can present local or systemic envenomation, while neurotoxicity and respiratory paralysis are the main cause of death. The mainstay of management is anti-snake venom (ASV), which is highly effective, but liable to cause severe adverse reactions including anaphylaxis. The types of adverse reaction to polyvalent anti-snake venom have not been previously studied in Bangladesh. In this prospective observational study carried out between 1999 and 2001, in the Snake Bite Study Clinic of Chittagong Medical College Hospital, 35 neurotoxic-snake-bite patients who had received polyvalent anti-snake venom were included while the ones sensitized to different antitoxins and suffering from atopy were excluded. The common neurotoxic features were ptosis (100\%), external ophthalmoplegia $(94.2 \%)$, dysphagia $(77.1 \%)$, dysphonia $(68.5 \%)$ and broken neck sign $(80 \%)$. The percentage of anti-snake venom reaction cases was $88.57 \%$; pyrogenic reaction was $80.64 \%$; and anaphylaxis was $64.51 \%$. The common features of anaphylaxis were urticaria (80\%); vomiting and wheezing (40\%); and angioedema (10\%). The anti-snake venom reaction was treated mainly with adrenaline for anaphylaxis and paracetamol suppository in pyrogenic reactions. The average recovery time was 4.5 hours. Due to the danger of reactions the anti-snake venom should not be withheld from a snakebite victim when indicated and appropriate guidelines should be followed for its administration.
\end{abstract}

KEY WORDS: snakebite, anti-snake venom, adverse reaction, anaphylaxis.

CONFLICTS OF INTEREST: There is no conflict.

\section{CORRESPONDENCE TO:}

MOHAMMAD ROBED AMIN, Consultant of Medicine, Department of Medicine, Hathazari Upazilla Health Complex, Chittagong, Bangladesh, 28 R. C. Church Road, Patherghata, Chittagong, Bangladesh. Email: robedamin@yahoo.com. 


\section{INTRODUCTION}

Snakebite is an important occupational health hazard which may lead to death in rural areas of many tropical countries including Bangladesh. An epidemiological study, in Bangladesh, estimated 4.3 snakebites per 100,000 humans, with a mortality rate of $20 \%(18)$. There are 82 species of Bangladeshi snakes among which 28 are venomous (8) and present clinical importance; these species include varieties of cobras, kraits, Russell's vipers, green and sea snakes. Cobra and krait bites are associated with neurotoxicity, presenting or not local tissue necrosis $(6,1)$. Usually, the common serious neurotoxic features comprise ptosis, external ophthalmoplegia, dysphagia, dysphonia, loss of tendon reflexes and respiratory paralysis $(5,16,17$, 19). The wellspring of venomous bite management is anti-snake venom (15), a protein product, liable to cause adverse reaction that may be immediate or delayed. The immediate reaction consists of anaphylaxis, characterized by urticaria, sweating, bronchospasm, laryngospasm, hypotension, shock, coma and even death. The early reaction comprises pyrogenic response characterized by chills, shivering, goosebumps and fever, while the delayed version involves serum sickness characterized by fever, rash, lymphadenopathy and arthralgia. Antivenoms may be withheld by physicians despite their clear indication due to the belief that they are more dangerous than the disease. This idea remains a vestige of an earlier epoch, when antivenoms were very crude and many patients had been previously sensitized by the use of other antisera. The acute adverse reaction to anti-snake venom, which includes severe anaphylaxis and is commonly followed by pyrogenic reaction, has been a point of concern to several physicians (13). The timely approach to manage these reactions with adrenaline, steroids, phenothiazines and paracetamol creates a simple solution (14). The severity of a venomous bite is more dangerous than the antivenom if it remains untreated while active management of venomous snakebites through proper guidelines should be practiced without fear.

\section{MATERIALS AND METHODS}

The present study aimed to document the use of and the adverse reactions to antisnake venoms. It was carried out over a period of two years from May 1999 to June 2001 in the Snake Bite Study Clinic (SBSC) of the Chittagong Medical College Hospital $(\mathrm{CMCH})$. The patients that had one or more neurological manifestations after a snakebite, treated by anti-snake venom (ASV), were included in this study. 
The persons that presented previous neurological illness were excluded, as were the allergic or atopic ones. Persons previously sensitized with antisera (tetanus or diphtheria) and that had had pre-hospital management, in the community, by a local physician - with adrenaline, antihistamine or steroids - were also excluded from this particular series. Detailed neurological examination was performed and the patients were monitored for at least five days in every case. The routine tests were: blood exam, platelet count, microscopic urine examination, ECG and 20-minute-wholeblood clotting test. Peak expiratory flow rate was measured in enrolled patients, except for those who were not able to cooperate or those who needed artificial ventilatory support during presentation. All patients were treated with Polyvalent Haffkine $\AA$ anti-snake venom, manufactured by Haffkine Bio-Pharmaceuticals Company (India) as is Snake Antivenin ${ }^{\circledR}$ (lyophilized polyvalent enzyme refined equine immunoglobulin).

The patients who were treated with anti-snake venom also received anticholinesterase neostigmine (subcutaneously) and atropine (intravenously). Standby preparation included adrenaline, chlorpheniramine, hydrocortisone, oxygen, salbutamol nebulization, cardiac monitor, ambo bag, and facility for mechanical ventilation. Patient recoveries were monitored for anti-snake venom reaction. Anaphylaxis was observed at bedside for one hour, during the whole period of administration, and also subsequently, up to 180 minutes, in cases of pyrogenic reaction. The anaphylaxis and the pyrogenic reaction were actively managed according to the World Health Organization South-East Asia Regional Office (WHO/SEARO) guideline for snakebite management (10). The patients who had adverse reaction were observed during recovery. The data analysis was performed in EPI Info 6 statistical packages. Means ( \pm standard error of the mean), median (range), numbers and percentages were used to describe the parameters.

\section{RESULTS}

During the study period, between May 1999 to June 2001, 537 snakebite victims were admitted in the Chittagong Medical College Hospital. Among these patients, most bites were by non-venomous animals (365).

One hundred and seventy-two cases involved venomous reptiles, most frequently green snakes $(60 \%)$, followed by cobras $(29 \%)$ and the remainder by kraits $(11 \%)$. Sixty-three patients presented neurotoxic features with or without local 
envenomation; among this group, 35 cases were included in this series. The selection was based on neurotoxicity presence and absence of previous antiserum use or prior use of prophylactic drugs during pre-hospital or hospital management. The 35 enrolled patients received ASV during the study period.

\section{Age}

Studied patient ages ranged from 3.5 to 85 years old (mean 27.5). Most victims were between 6 and 30 years (70\% of total patients) (Table 1$)$.

Table 1. Age distribution of neurotoxic snakebites $(n=35)$

\begin{tabular}{c|c|c}
\hline $\begin{array}{c}\text { Age } \\
\text { (years) }\end{array}$ & $\begin{array}{c}\text { Number of } \\
\text { patients }\end{array}$ & Percentage \\
\hline $1-5$ & 1 & 2.8 \\
\hline $6-10$ & 6 & 17.1 \\
\hline $11-20$ & 11 & 31.4 \\
\hline $21-30$ & 8 & 22.8 \\
\hline $31-40$ & 4 & 11.4 \\
\hline $41-50$ & 2 & 5.7 \\
\hline$>50$ & 3 & 8.6 \\
\hline
\end{tabular}

\section{Sex}

Of the 35 enrolled patients, 19 were male (54.2\%) and 16 were female (45.8\%).

\section{Clinical Feature: Neurological Manifestation}

The most common neurological manifestation observed in snakebite victims was ptosis, in various degrees, which was found in all patients. The next ordinary feature was external ophthalmoplegia. Other signs were dysphagia, dysphonia, broken neck sign (paralysis of the cervical flexor muscles), hand-grip weakness, depressed reflexes and generalized weakness (Figure 1) 


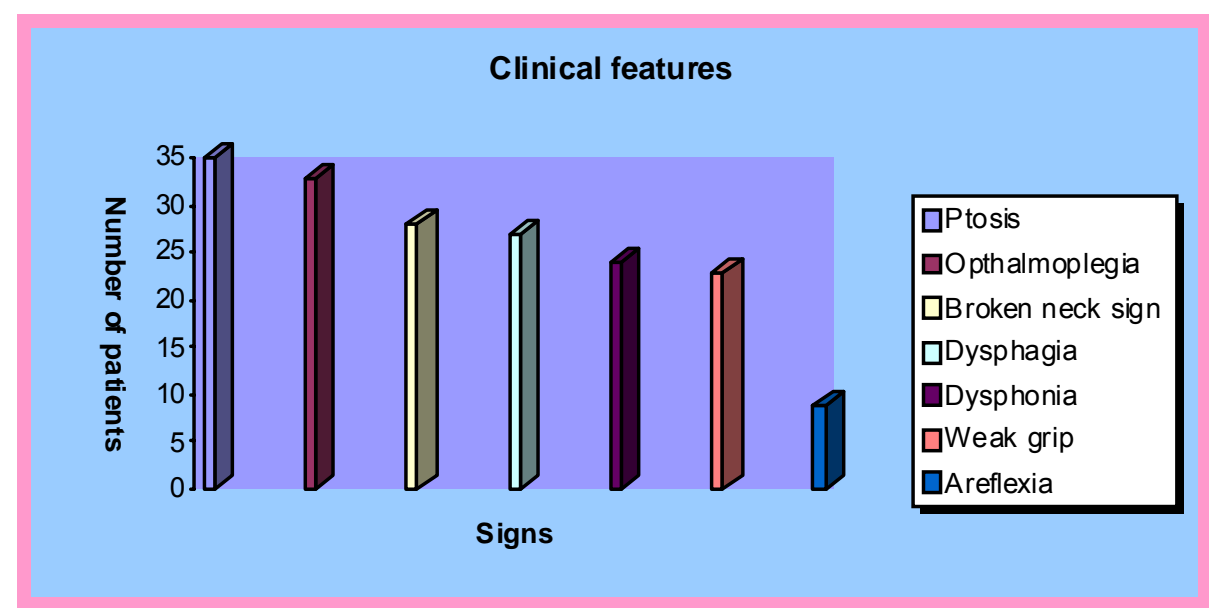

Figure 1. Different features of neurological manifestations $(n=35)$.

\section{Investigation}

The total count (TC) of white blood cells (WBC) in patients presenting neurotoxic signs showed neutrophilic leukocytosis in 23 of them (TC was more than 1,1000 and neutrophils $>75 \%$ ) (Table 2). Urinary albumin was found in 19 cases (54\%). Bedside 20-minutes-whole-blood clotting test was normal in all cases, indicating no coagulation abnormality. ECG was abnormal only in two patients; one showed ST depression and $T$ inversion in lead $I, A V_{L}$ and $V_{5-6}$; and the other showed $T$ wave inversion in all the chest leads.

Table 2. Investigation of neurotoxic snakebites $(n=35)$

\begin{tabular}{c|c|c}
\hline Investigation & $\begin{array}{c}\text { Number } \\
\text { of patients }\end{array}$ & Percentage \\
\hline Polymorph leukocytosis & 23 & 65.7 \\
\hline Urinary albumin & 19 & 54.2 \\
\hline Abnormal ECG & 2 & 5.7 \\
\hline 20 min.WBCT abnormal & 0 & 0 \\
\hline
\end{tabular}




\section{Treatment}

All the selected patients were treated with polyvalent anti-snake venom and most of them received single doses (10 vials).

\section{Auxiliary Treatment}

Along with the antivenom, anticholinesterase neostigmine was given to all patients. Five persons required artificial ventilatory support.

\section{Anti-snake Venom Reaction}

In the current study, $88.5 \%$ of the patients developed mild to severe forms of antisnake venom reaction. Only four of them, of the 35 , did not present any early or delayed adverse response.

\section{- Type of anti-snake venom reaction}

The victims that received antivenom treatment presented the following reactions: antivenom reaction (31 patients), early anaphylactic response (20 patients), pyrogenic reaction (25 patients) and both anaphylactic and pyrogenic reactions (14 patients).

\section{- Anaphylactic reaction}

Of the 35 enrolled patients, 20 developed anaphylactic reaction. Most cases presented urticaria with itching (Figure 2). The next common symptoms were nausea and vomiting, followed by severe anaphylaxis with wheezing and rhonchi. Only two patients developed angioedema. The other presentations were cough, headache, fever, tachycardia and palpitation. 


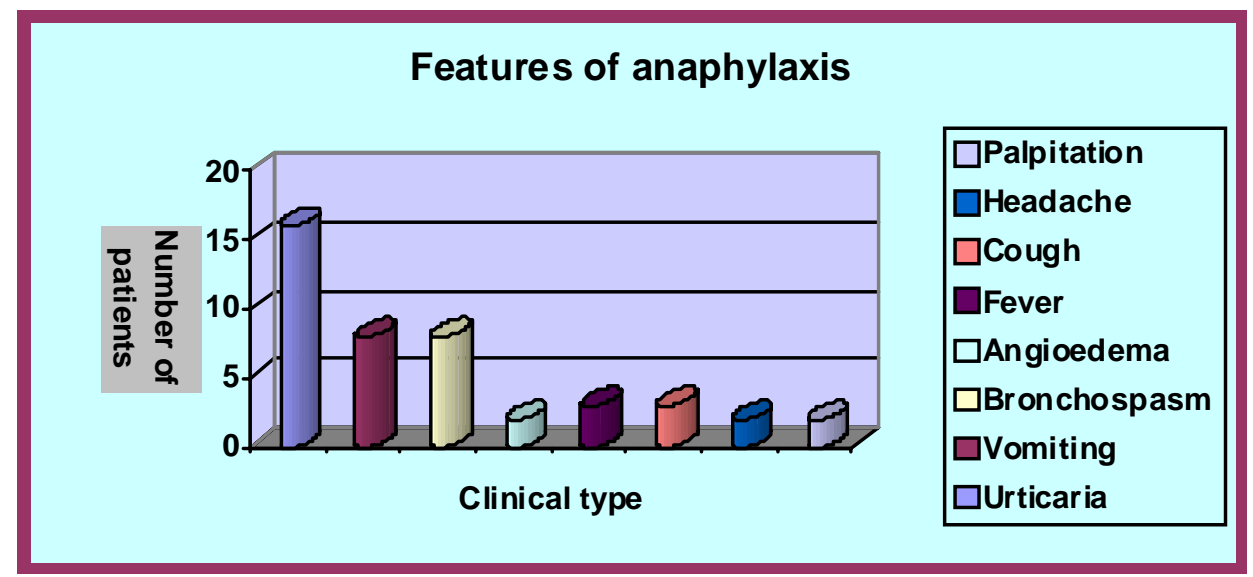

Figure 2. Clinical presentations of anaphylaxis.

\section{- Onset of anaphylactic reactions}

The onset of the reactions varied from 10 to 50 minutes, with an average time of 28 minutes. Interestingly, the antivenom infusion in this study did not produce anaphylactic reaction beyond one hour. The antivenom dose also ranged from 25 to $100 \mathrm{~mL}$, with an average of $55 \mathrm{~mL}$ (Figure 3).

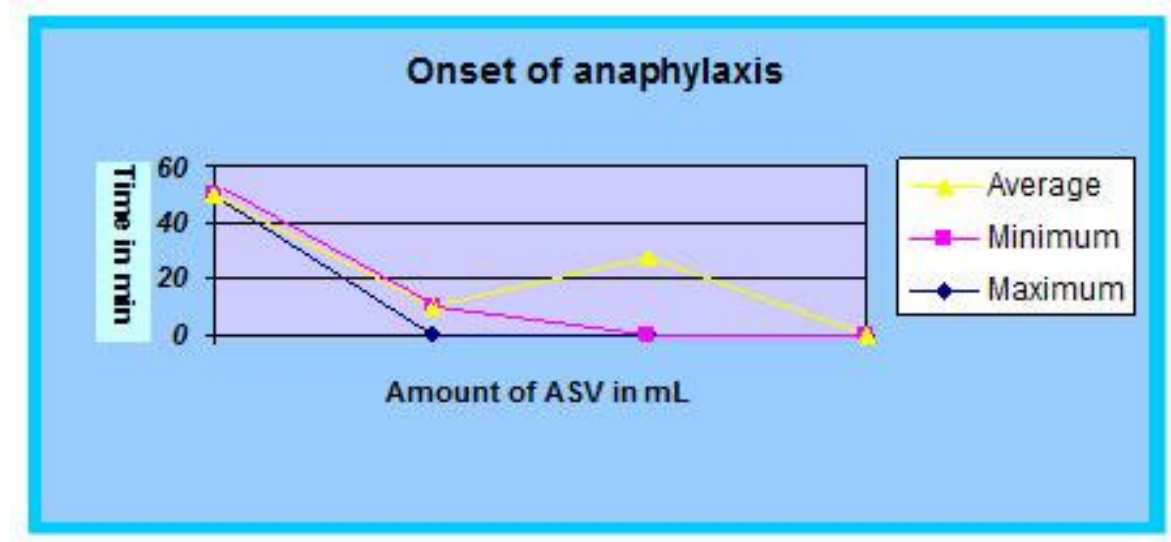

Figure 3. Onset of anaphylaxis $(n=20)$.

\section{- Management of antivenom reaction}

In this series, 25 patients developed pyrogenic reaction while 20 had anaphylactic one. The pyrogenic response was treated with paracetamol suppository, with $100 \%$ recovery. The anaphylactic reaction was managed with adrenaline (intramuscular 
injection), hydrocortisone (intravenous injection), antihistamine chlorpheniramine (intravenous injection) and ranitidine (intravenous injection). Of these 20 patients, only two needed two or more adrenaline doses to recover from anaphylaxis.

\section{- Outcome of treatment}

All 35 cases of snakebites with neurotoxic manifestations completely recovered without neurological sequelae. There was no death in the studied group.

\section{DISCUSSION}

The age of the victims ranged from 3.5 to 85 years old and among the 35 selected cases, $71 \%$ of them were in the 6 to 30 -year-age group. Only three persons $(8.6 \%)$ were older than 50 years. The present observation is similar to other data, for example, in a hospital based study, conducted in northern Bangladesh, the mean age was 26.7 years (7) and Devkota et al. (4) found, in Nepal, that $85 \%$ of the victims were younger than 45 years old. Thus, it was observed that snakebites occur especially among active workers. In the present study, there was a slightly higher number of male $(54.2 \%)$ than female patients $(48.2 \%)$, which is not consistent with similar studies - conducted in Rajshahi, Bangladesh (7), and in Nepal (4) - that found exclusively male victims outside home during working hours.

The first universal sign of neurotoxicity found in the present study was incomplete or complete ptosis with external ophthalmoplegia, which was also observed by Mitrakull et al. (9) and by Campbell (2). Broken neck sign followed by dysphagia and dysphonia were other neurological signs. Only five cases required artificial ventilatory support due to respiratory muscle paralysis. The great involvement of neck muscle explained the traditional belief that there is a sequential muscle paralysis (2); however, this cannot be true since several muscle groups are frequently involved together $(3,12)$.

All enrolled patients were treated with the recommended dose of polyvalent antisnake venom according to WHO/SEARO guidelines for the clinical management of snakebites. A study, accomplished in northern Bangladesh, registered seven deaths (26\%) among victims treated with anti-snake venom doses ranging from $20 \mathrm{~mL}$ to 40 $\mathrm{mL}$ (7). A prospective study of hospital practice in the Gampaha district, Sri Lanka, documented only $0.43 \%$ mortality when at least ten vials $(100 \mathrm{~mL})$ of antivenom were initially given to victims (11). These findings agree with Theakston et al. (15), who 
concluded that is prudent to give initial doses greater than $100 \mathrm{~mL}$ to patients with neurotoxic signs, as well as is necessary to repeat this dose every few hours, up to a total of at least $300 \mathrm{~mL}$, if there is no response.

The present results justify the antivenom use in an adequate initial amount and, if required, more doses can also be given to victims of neurotroxic snakebites that present poor response. The 35 cases of neurotoxic bites, in the current study, also received anticholinesterase neostigmine as an auxiliary treatment. Five of these persons presented respiratory muscle paralysis and, consequently, needed artificial ventilatory support.

Although the specific therapy for neurotoxic snakebite is antivenom, the drug efficacy may occasionally vary. Theakston et al. (15) emphasize the importance of anticholinesterases as an auxiliary treatment in victims of neurotoxic bites and they also concluded that Tensilon test is recommended in every case. Tensilon test was not performed in the current study due to its non-availability at Bangladesh market during the assay period. Unfortunately most patients $(88.57 \%)$ in this series developed early antivenom reaction. This rate is quite elevated concerning polyvalent anti-snake venom by Haffkine Bio-Pharmaceuticals Company (India), provided by Bangladesh Government. In Sri Lanka, a research found that $55.4 \%$ of patients who had received anti-snake venom were affected by adverse reactions and that there was no significant difference among reactions whether or not premedication was given (11). Sutherland (14), in a study accomplished in Australia, observed that polyvalent antivenoms were involved in $79 \%$ of recorded reaction cases, whereas monospecific ones were implicated in only $12.9 \%$. This statement justifies the use and importance of monospecific antivenoms over polyvalent ones (14).

In the present survey, 20 patients (64.5\%) suffered early anaphylactic reactions; while in the aforementioned Australian investigation only $12.9 \%$ of patients presented such problem (five of them had the immediate anaphylactic response) (14). Based on the latter results, it was concluded that patients who received polyvalent antivenom have a $10 \%$ chance of delayed reaction whereas immediate reactions may occur only when the combination of premedication and slow infusion is not used (14). Seneviratne et al. (11) analyzed 28 patients in Sri Lanka and observed that $50 \%$ of them had anaphylactic reactions as well as $63 \%$ developed pyrogenic responses that occurred when diluent water employed was other than that one provided by manufacturer. Therefore, it is possible to deduce that both anaphylactic and 
pyrogenic reactions are common after administration of polyvalent anti-snake venoms.

The most common presentation of anaphylactic reaction in the present survey was urticaria (16 cases) that comprised $80 \%$ of all anaphylactic reactions. The investigations carried out in Sri Lankan (11) and Australia (14) also registered urticaria as a common presentation.

The time interval between the ASV initial dose and the onset of symptoms differed very much in the current study. The earliest response was recorded 10 minutes after the ASV administration $(25 \mathrm{~mL}$ ) while the delayed one occurred 50 minutes later (100 $\mathrm{mL}$ ). The average interval observed was 28 minutes. Theakston et al. (15) also found $33 \%$ cases of anaphylactic responses 50 minutes after the initial ASV dose. In the present study, eighteen patients that had presented anaphylactic reactions were managed by a single dose of intramuscular adrenaline while two patients required second or third doses. Although concomitant intravenous antihistamine and intravenous hydrocortisone were administered in all cases, the response was more dramatic when adrenaline was given, which suggests that this substance may be employed as premedication. In a double-blind placebo-controlled trial, conducted in Polonnaruwa, Sri Lanka, the same ASV was utilized and it was established that premedication with adrenaline significantly reduces the risk of acute adverse reactions to antivenom sera (10). All snakebite patients of this series completely recovered, without any residual neurological sequelae. Even though five patients were in critical state when they arrived at the hospital, prompt anti-snake venom and auxiliary treatments in addition to quick management of antivenom reaction saved their lives. The effects of antivenom reactions were also impressive and no death occurred due to acute anaphylactic reaction. Given that, the antivenom use is justifiable whenever indicated without alarm; in the same manner, adverse reactions are rather simple to manage with available drugs.

\section{CONCLUSIONS}

The anti-snake venom is the specific antidote for venomous snakebites. In Bangladesh, polyvalent anti-snake venom by Haffkine Bio-Pharmaceuticals Company (India) is available in limited quantity. Although the antivenom is effective and specific, it presents a potential risk of adverse reactions including anaphylaxis. The antivenom must be administered in standard doses and routes to reach its 
optimum efficacy. Neurotoxic snakebites should be treated with anticholinesterases and artificial ventilatory support with all monitoring facilities available in health centers. The potential dangers of anaphylactic reactions - urticaria, vomiting, bronchospasm, hypotension, circulatory collapse and angioedema - can be managed with adrenaline, hydrocortisone, antihistamine and ranitidine. Due to the danger of reactions the anti-snake venom should not be withheld by physicians from a snakebite victim, when indicated and appropriate guidelines should be followed for its administration. The present study proved that anti-snake venom is effective and an eventual antivenom reaction can be easily managed through a proper approach.

\section{ACKNOWLEDGEMENTS}

The authors are grateful to the health professionals of studied hospitals for their kind support in managing the cases.

\section{ETHICAL APPROVAL}

The present study was approved by the Ethical Review Committee of the $\mathrm{CMCH}$. All patients gave written or verbal consent.

\section{REFERENCES}

1. BARFARAZ A., HARVEY AL. The use of chick biventer cervicis preparation to assess the protective activity of six international reference antivenoms on the neuromuscular effects of snake venoms in vitro. Toxicon, 1994, 32, 267-72.

2. CAMPBELL CH. A clinical study of venomous snake bite in Papua. Sydney: The University of Sydney, 1969. 208p. [MD Thesis].

3. CAMPBELL $\mathrm{CH}$. Venomous snake bite in Papua and its treatment with tracheostomy, artificial respiration and antivenin. Trans. R. Soc. Trop. Med. Hyg., 1964, 58, 263-73.

4. DEVKOTA UN., STEINMANN JP., SHAH LN. Snakebite in Nepal: a study from Sihara district, Nepal. J. Nep. Med. Assoc., 2000, 39, 203-9.

5. FAIZ MA., RAHMAN MR., DAS JC., MOLLAH AS., HUSSAIN MM. Neurotoxicity and tissue necrosis following bite by cobra (Naja) in Chittagong, Bangladesh. Proc. XIV Int. Cong. Trop. Med. Malar., 1996, 52, 282. 
6. HARVEY AL., BARFARAZ A., THOMSON E., FAIZ A., PRESTON S., HARRIS JB. Screening of snake venoms for neurotoxic and myotoxic effects using simple in vitro preparations from rodents and chicks. Toxicon, 1994, 32, 257-65.

7. ISLAM QT., FAIZ MA., AZHAR MA., EKRAM ARMS., ALAM MT. Snake bite in northern Bangladesh: a hospital based study of 68 cases. TAJ, 1999, 12, 135-8.

8. KHAN MAR. Sareesrip Prani. In: KHAN MAR. Bangladesher Bannya Prani. Dhaka: Bangla Academy, 1987, 42,168.

9. MITRAKULL C., DHAMKRONG-AT A., FUTRAKUL P., THISYAKORN C., VONGSRISART K., VARAVITHYA C., PHANCHAROEN S. Clinical features of neurotoxic snake bite and response to antivenom in 47 children. Am. J. Trop. Med. Hyg., 1984, 33, 1258-66.

10. PREMAWARDENA AP., DE SILVA CE., FONESKA MMD., GUNATILAKE SB., DE SILVA HJ. Low dose subcutaneous adrenaline to prevent acute adverse reactions to antivenom serum in patients bitten by snakes: a randomized placebo controlled trial. Br. Med. J., 1999, 318, 1041-3.

11. SENEVIRATNE SL., WICKRAMA WSS., OPANAYAKA CJ., RATNAYAKE NSL., GUNATILAKE SB., DE SILVA HJ. Use of antivenom serum in snake bite: a prospective study of hospital practice in the Gampaha district. Ceylon Med. J., 2000, 45, 65-8.

12. SETHI PK., RASTOGI JK. Neurological aspects of ophitoxemia (Indian krait): a clinic-electromyographic study. Indian J. Med. Res., 1981, 73, 269-76.

13. SUTHERLAND SK. Acute untoward reactions to antivenoms. Med. J. Aust., 1997, 2, 841-2.

14. SUTHERLAND SK. Antivenom use in Australia. Premedication, adverse reactions and the use of venom detection kits. Med. J. Aust., 1992, 157, 734-9.

15. THEAKSTON RD., PHILLIPS RE., WARRELL DA., GALAGEDERA Y., ABEYSEKERA DT., DISSANAYAKA P., DE SILVA A., ALOYSIUS DJ. Envenoming by the common krait (Bungarus caeruleus) and Sri Lankan cobra (Naja naja naja): efficacy and complications of therapy with Haffkine antivenom. Trans. R. Soc. Trop. Med. Hyg., 1990, 84, 301-8.

16. THEAKSTON RD., REID HA. Development of simple standard assay procedures for the characterization of snake venom. Bull. World Health Organ., 1983, 61, 94956. 
17. WARELL DA. Snake venoms in science and clinical medicine. 1. Russell's viper: biology, venom and treatment of bites. Trans. R. Soc. Trop. Hyg., 1989, 83, 732-40. 18. WORLD HEALTH ORGANIZATION. Progress in the characterization of venoms and standardization of antivenoms. Geneva: WHO Offset Publishers 58, 1981. 23-3p. 19. WUSTER W., THORPE RS. Asiatic cobras: systematics and snakebite. Cell. Moll. Life Sci., 1991, 47, 205-9. 